\title{
XÂY DỰNG BÀI GIẢNG THEO CHUẦN ĐẦU RA CDIO NHẰM PHÁT TRIỂN KỸ NĂNG THIẾT KẾ VÀ VẬN HÀNH CÁC HỆ THỐNG ĐIỀU KHIỂN TỬ ĐộNG
}

\author{
Nguyễn Thị Huệ $\hat{(}^{(*)}$
}

(*) Thạc sĩ. Truòng Đại học Công nghệ Đồng Nai. Email: nguyenthihue@dntu.edu.vn.

DOI: $10.37550 /$ tdmu.CFR/2021.01.103

\section{Tóm tắt}

Trong bài báo này đề cập đến phương pháp xây dựng bài giảng môn Tự động hóa quá trình công nghệ dành cho sinh viên chuyên ngành Kỹ thuật Điện - Điện tư của Truờng Đại học Công nghệ Đồng Nai (DNTU). Mục tiêu chính của môn học là nhấn mạnh vào các kĩ năng: Làm việc nhóm, làm việc độc lập, cách giải quyết vấn đề, thiết kế và vận hành các hệ thống điều khiển tụ động. Bài giảng được xây dụng dưa trên việc phân chia các nhóm sinh viên (mối nhóm gồm có 5 sinh viên) thực hiện trên bài tập lớn được giao ở trong phòng thí nghiệm. Cách thực hiện bài tập trong phòng thí nghiệm của sinh viên: Truớc tiên sinh viên phải có một bài giới thiệu, trong bài giới thiệu nêu rõ: Cách giải quyết vấn đề, công tác chuẩn bị công việc, cách làm việc độc lập trong phòng thí nghiệm, cách làm việc nhóm. Sau đó sẽ là một buổi báo cáo tổng kết. Ngoài ra, có một dụ án nhỏ mà nhiệm vu của sinh viên là viết hướng dẫn quy trình sủ dụng cho người vận hành. Để làm được điều này yêu cầu sinh viên phải hiểu biết sâu về hệ thống điều khiển PLC trong công nghiệp (Bộ điều khiển lập trình logic), phát triển giao diện điều khiển người máy và HMI, cảm biến công nghiệp, các cơ cấu chấp hành.

Tù̀ khóa: lập trình PLC, tụ động hóa, tiêu chuẩn CDIO, kỹ năng làm việc nhóm

\section{1. Đặt vấn đề}

Theo khung CDIO, mục tiêu của đào tạo chuyên ngành Kỹ thuật Điện - Điện tử của trường DNTU là mọi kỹ sư tốt nghiệp phải có khả năng: Nhận biết - Thiết kế - Thực hành - Vận hành các sản phẩm, lập được quy trình kỹ thuật có độ gia tăng phức tạp và vận hành được các hệ thống trong một môi trường hiện đại, kỹ năng làm việc độc lập, kỹ năng làm việc nhóm.

Theo cách học truyền thống, một khóa học về kỹ thuật sẽ được bắt đầu với các môn cơ sở lý thuyết (bao gồm các công cụ phân tích), sau đó mới đến phương pháp thiết kế. Bài kiểm tra cuối kỳ thường là bằng tự luận, vấn đáp hay trắc nghiệm, việc kiểm tra cuối kỳ bằng hình thức thực hành hiếm khi được thực hiện. Việc thực hiện giảng dạy theo phương pháp truyền thống làm sinh viên dễ tiếp thu kiến thức một cách thụ động, giờ học cũng 
buồn tẻ và kiến thức chỉ thiên về lý thuyết. Bởi vì không có nhiều cơ hội thực hành, nên học sinh khó có thể áp dụng những kiến thức đã học vào thực tiễn.

Xây dựng bài giảng theo chuẩn đầu ra CDIO của môn học là nhấn mạnh về giai đoạn Triển khai và Vận hành trong quá trình tạo ra một sản phẩm hoặc một hệ thống.Tuy nhiên, để làm được điều đó ta cần phải cung cấp cho sinh viên một lượng lý thuyết tối thiểu cần thiết và một thách thức đặt ra trong giảng dạy là việc sinh viên DNTU sử dụng toán học còn rất hạn chế

Bên cạnh đó với mục tiêu là nhấn mạnh các giai đoạn Triển khai và Vận hành nhằm tạo cho sinh viên hứng thú và tích cực học tập. Cốt lõi của việc thực hiện và kiểm tra khóa học bao gồm việc yêu cầu sinh viên làm bài tập lớn trong phòng thí nghiệm và một dự án nhỏ. Tuy nhiên vướng mắc đặt ra là việc lập kế hoạch làm việc cho sinh viên, vì thiết bị trong phòng thí nghiệm và giảng viên hướng dẫn còn hạn chế, chưa đảm bảo hết yêu cầu cho sinh viên.

\section{Về môn học}

Tư động hóa quá trình công nghệ là một môn học quan trọng trong quy trình sản xuất sản phẩm và nhiệm vụ đối với cơ chế điều khiển tự động là tạo ra sản phẩm, quy trình,hoặc hệ thống hoạt động theo cách mong muốn. Lĩnh vực này đôi khi được gọi là công nghệ ẩn vì sự hiện diện của nó trong các ứng dụng khác nhau hiếm khi được nhìn thấy. Thay vào đó các hiệu ứng có thể được quan sát gián tiếp thông qua hoạt động của đối tượng được kiểm soát. Tự động kiểm soát có thể được tìm thấy trong hầu hết các ứng dụng, từ ngành công nghiệp chế biến, ứng dụng hàng không vũ trụ, xe du lịch và xe tải, hệ thống điện, các sản phẩm tiêu dùng như điện thoại di động và máy tính, thiết bị kỹ thuật y sinh... Các mục tiêu cho việc sử dụng các hệ thống điều khiển tự động phụ thuộc vào ứng dụng, nhưng chúng liên quan đến các khía cạnh như chất lượng, năng suất, an toàn, sử dụng hiệu quả năng lượng và các tài nguyên khác, tiện nghi... Một trong những tính năng hấp dẫn của nó là việc tạo ra một hệ thống điều khiển thế giới thực bao gồm một số lĩnh vực như các mô hình và công cụ toán học, kiến thức quy trình, công nghệ phần cứng và phần mềm, công nghệ cảm biến và đo lường... các mô hình và các công cụ toán học trong một khóa học cơ bản về điều khiển tự động thường liên quan đến giải tích (phương trình vi phân và tích phân), đại số tuyến tính và phép biến đổi.

Môn học này được coi là một môn cốt lõi về chuyên ngành kỹ thuật Điện - Điện tử và nó là một phần của chương trình giảng dạy trong nhiều chương trình giáo dục kỹ thuật trên thế giới. Do đó có rất nhiều sách giáo khoa viết về môn học này. Và hầu hết các sách giáo khoa có cấu trúc tương tự, đầu tiên là một số ví dụ, tiếp theo là cơ sở lý thuyết về toán học, sau đó là tập trung trình bày về các phương pháp phân tích và thiết kế khác nhau.Mức độ khó dễ phân theo cấp độ của sinh viên. Vì vậy, điều này mang ý nghĩa đại diện cho cách trình bày về chủ đề trong giáo dục kỹ thuật. Một thách thức sư phạm trong giáo dục điều khiển tự động là kết nối thế giới của các mô hình toán học, tức là vi phân các phương trình và phép biến đổi Laplace, với thế giới vật lý thực. Bước đầu tiên thường là sử dụng mô phỏng để kích thích trực giác và sự tìm hiểu của sinh viên, đồng thời giúp sinh viên kết nối 
thuộc tính miền thời gian, tần số và bước thứ hai là thực hiện thí nghiệm sử dụng các quá trình vật lý. Mặc dù trên thực tế là các thí nghiệm sử dụng phần cứng đóng một vai trò quan trọng nhưng vì lý do về mặt kinh tế, xu hướng sử dụng ngày càng nhiều thiết bị ảo, phần mềm và từ $\mathrm{xa}$

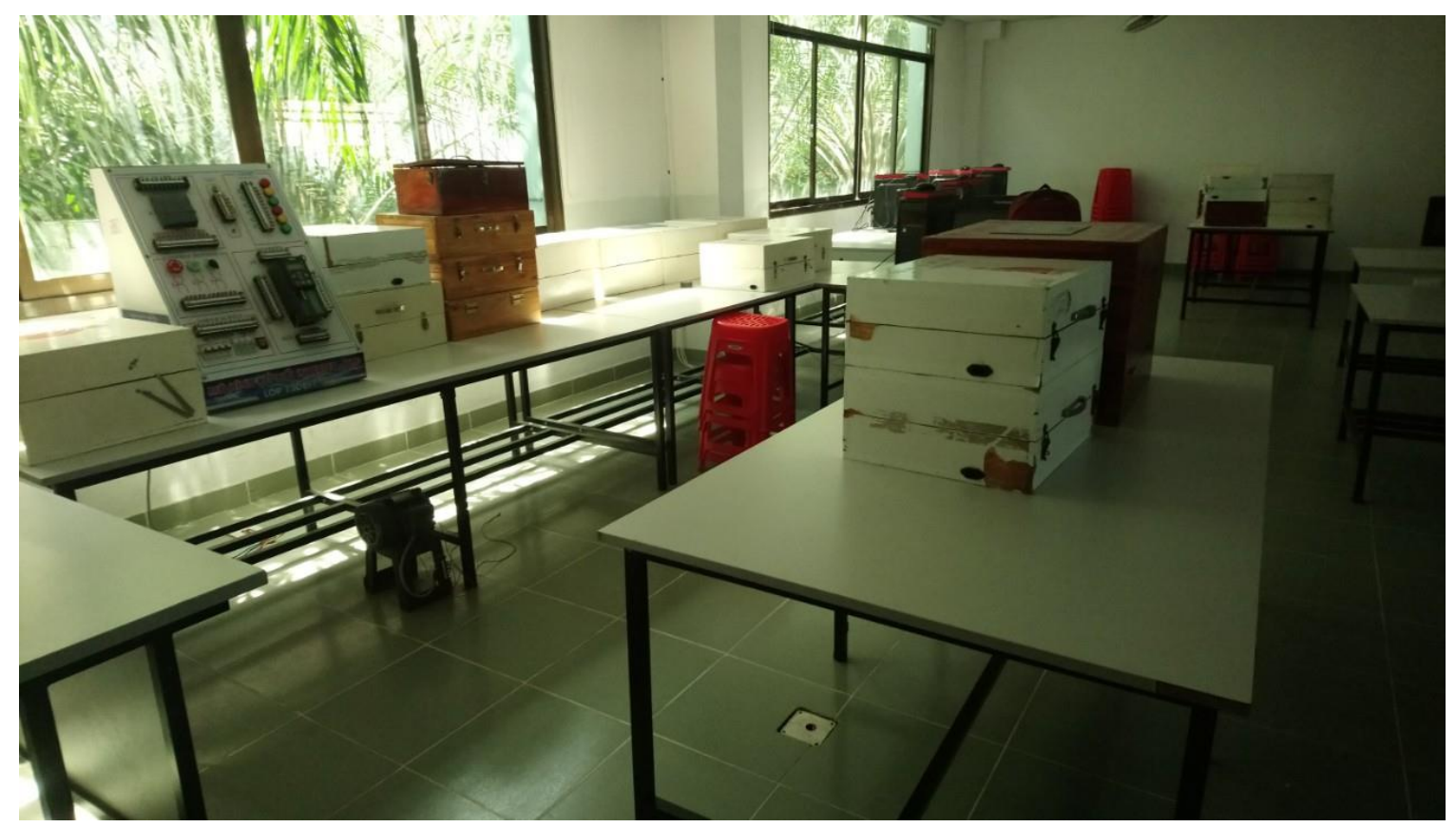

Hinh 1. Phòng thí nghiệm mạng truyền thông công nghiệp

\section{Về bối cảnh}

\subsection{Thông tin co bản}

Khóa học được cung cấp cho chương trình đào tạo kỹ sư chuyên ngành Kỹ thuật Điện - Điện tử học kỳ 2 của năm thứ 2 của trường DNTU. Môn học gồm 3 tín chỉ và thường có khoảng 50 sinh viên trong một lớp học.

\subsection{Phưong pháp thục hiện}

Mục tiêu chính của môn học: Nhận biết - Thiết kế - Thực hành - Vận hành các sản phẩm, lập được quy trình kỹ thuật có độ gia tăng phức tạp và vận hành được các hệ thống trong một môi trường hiện đại, kỹ năng làm việc độc lập, kỹ năng giải quyết vấn đề...

Các bước thực hiện:

Bước 1: Giảng viên sẽ giới thiệu cho sinh viên về cách thức thực hiện môn học theo chuẩn đầu ra CDIO.

Bước 2: Giảng viên sẽ có 4 bài giảng để hướng dẫn tập trung cho sinh viên ở trên lớp, nội dung của bài giảng liên quan đến những kiến thức lý thuyết của môn học. Song song với 4 bài giảng ở trên lớp là 4 bài thực hành trong phòng thí nghiệm. Thông thường giảng viên sẽ chia lớp thành 2 nhóm để vào phòng thí nghiệm, mỗi nhóm có khoảng tầm 25 
sinh viên. Nội dung bài thực hành liên quan đến những kiến thức trong phần lý thuyết và một phần sẽ hỗ trợ cho bài tập lớn trong phòng thí nghiệm và dự án nhỏ của sinh viên

Bước 3: Giảng viên sẽ chia nhóm sinh viên, mỗi nhóm có thể dao động từ 4 đến 6 sinh viên. Mỗi nhóm sẽ được làm một bài tập lớn trong phòng thí nghiệm và một dự án nhỏ

Bước 4: Sinh viên sẽ làm bài tập lớn trong phòng thí nghiệm của trường và thêm một dự án nhỏ. Các bài tập trong phòng thí nghiệm là yêu cầu sinh viên phải sử dụng được phần cứng và phần mềm liên quan đến môn học, bên cạnh đó phải kết hợp phần cứng và phần mềm để thực hiện các bài thí nghiệm về điều khiển hệ thống trong phòng thí nghiệm.

Bước 5: Sinh viên sẽ có một buổi báo cáo về cách thức làm việc của nhóm

Bước 6: Cuối cùng là một buổi báo cáo về bài tập lớn và dự án nhỏ của nhóm.

Dự án nhỏ là một bài tổng hợp nó kết nối chặt chẽ với bài thí nghiệm và nhiệm vụ của sinh viên là viết bài hướng dẫn sử dụng cho hệ thống điều khiển mà sinh viên vừa thực hiện trong bài tập lớn. Các bài tập trong phòng thí nghiệm là công việc nhóm của sinh viên, trong nhóm sẽ chia ra các phần nhỏ để mỗi sinh viên phải đảm nhận những công việc mà mình được giao và thời lượng cho mỗi bài tập trong phòng thí nghiệm có thể lên đến 4 tuần.

Để thực hiện được mục tiêu của môn học yêu cầu sinh viên phải nắm được kiến thức kỹ năng cần thiết từ các môn học trước trong chương trình giảng dạy bao gồm thứ tự đầu tiên phương trình vi phân và tích phân từ môn học giải tích, các kỹ năng cơ bản trong lập trình, kiến thức cơ bản về số nhị phân cùng với kỹ năng viết báo cáo. Bên cạnh đó sinh viên cần phải có khả năng làm việc một cách độc lập, kỹ năng làm việc nhóm và đưa ra các sáng kiến và sử dụng cách tiếp cận có hệ thống để xử lý sự cố.

Đồng thời môn học cũng đòi hỏi một số lượng đáng kể kế hoạch của sinh viên. Nếu sinh viên không biết cách lập kế hoạch một cách khoa học thì thông thường khi kết thúc khóa học sinh viên sẽ không hoàn thành được mục tiêu đề ra. Bởi vì hiện tại trường Đại học Công nghệ Đồng Nai chỉ có duy nhất 1 phòng thí nghiệm về tự động hóa, vì vậy yêu cầu sinh viên khi sử dụng phòng thí nghiệm phải đăng ký trước để có thể sắp xếp phòng cho sinh viên thí nghiệm và giảng viên hướng dẫn. Cũng cần lưu ý rằng các sinh viên theo dõi các môn học song song, trong đó một trong số chúng cũng bao gồm các bài tập trong phòng thí nghiệm.

\section{Phương pháp đánh giá}

Kết quả học tập theo chuẩn đầu ra CDIO được đánh giá cho từng thành viên theo các mục như sau:

\subsection{Kiến thức}

Sinh viên phải nắm được những kiến thức liên quan đến môn học, đồng thời có thể nắm vững và điều chỉnh được bộ điều chỉnh $\mathrm{PI}$, sử dụng được các phần cứng và phần mềm, hiểu được các chức năng của các bộ phận khác nhau của bộ điều khiển và cách điều chỉnh chúng. Đồng thời có thể vận hành được hệ thống. 


\subsection{Các kỹ năng cá nhân và nghề nghiệp}

Thang điểm sẽ được đánh giá theo mức độ thời gian hoàn thành bài tập lớn của từng cá nhân. Như đã đề cập, sinh viên vửa phải thực hiện các bài tập trong phòng thí nghiệm, đồng thời quản lý song song với các môn học khác trong cùng một thời điểm vì vậy việc lên kế hoạch cho việc thực hiện bài học hết sức quan trọng và đòi hỏi sinh viên phải thực hiện một cách hết sức nghiêm túc.

Điểm cho việc tích cực trong công việc, bao gồm: sinh viên lên kế hoạch gặp gỡ giảng viên để trình bày về công việc thực hiện của mình, sinh viên tiếp thu những ý kiến đóng góp của giảng viên và việc sinh viên hăng hái tham gia đóng góp ý kiến nhóm

\subsection{Kỹ năng giao tiếp giữa các cá nhân và kỹ năng làm việc nhóm}

Kỹ năng giao tiếp giữa các cá nhân: Khả năng làm việc được đánh giá gián tiếp thông qua khả năng giải quyết nhiệm vụ cùng nhau trong phạm vi giới hạn về thời gian của hai thành viên trong nhóm. Hơn nữa, yêu cầu cả hai sinh viên trong nhóm phải giải thích cách giải quyết vấn đề của nhóm và cách tạo ra các hoạt động trong nhóm.

Kỹ năng làm việc nhóm: Được đánh giá bằng cách sinh viên phải thuyết phục được các thành viên trong nhóm đồng tình với ý kiến của mình. Bên cạnh đó sinh viên phải có khả năng tổ chức công việc, khả năng kiểm soát tình huống cùng với sự quyết tâm trong công việc...

Giao tiếp văn bản được đánh giá thông qua dự án nhỏ là viết hướng dẫn sử dụng cho bài thí nghiệm của nhóm.

\subsection{Hình thành, thiết kế, triển khai và vận hành các hệ thống:}

Điểm mấu chốt của phần này nhấn mạnh vào việc sinh viên phải thực hiện được các giai đoạn của quy trình kỹ thuật. Có thể phát triển một hệ thống điều khiển bao gồm cả giao diện điều hành, đáp ứng các yêu cầu liên quan đến chức năng sử dụng phần cứng công nghiệp thực như bằng chứng là các kết quả học tập đã đạt được

\subsection{Sinh viên đánh giá lẫn nhau}

Phương pháp đánh giá như sau: Thành lập một bảng đánh giá của các thành viên trong nhóm. Các thành viên trong nhóm sẽ tự đánh giá lẫn nhau (đánh giá kín) về mức độ hoàn thành, mức độ tích cực, mức độ hợp tác, ý tưởng, sự kiên trì, tinh thần trách nhiệm, tôn trọng bạn bè...

\section{Kết quả và thảo luận}

- Với việc học tập dựa trên chuẩn đầu ra CDIO cho phép sinh viên học tập một cách chủ động hơn, đồng thời tạo được động lực giúp sinh viên học tập tích cực, sinh viên có thể phát huy được những thế mạnh của mình.

• Bên cạnh đó, những thay đổi khác, chẳng hạn như việc sử dụng rộng rãi phương pháp đánh giá khác như so sánh, hỏi ý kiến và thảo luận phản hồi về quy trình của nhóm đã làm nổi bật mối tương quan tích cực giữa các thành viên trong nhóm 
• Việc viết sách hướng dẫn vận hành giúp đào tạo tốt về giao tiếp bằng văn bản.

- Các hướng dẫn, ví dụ về danh sách kiểm tra, liên quan đến quá trình viết có thể được cải thiện

- Việc tổ chức và thực hiện khóa học kiểu này đòi hỏi phải có sự tham gia giảng dạy của giảng viên cần quan tâm đầy đủ đến việc lập kế hoạch và thời gian của các hoạt động học tập khác nhau.

• Tương tự, khóa học yêu cầu sinh viên phải lập kế hoạch cẩn thận để phù hợp với nhiều hoạt động học tập vào lịch trình cùng với các khóa học khác.

- Các buổi trợ giúp rất quan trọng để đảm bảo rằng học sinh chuẩn bị đầy đủ trước khi thực hiện các bài tập trong phòng thí nghiệm.

- Một tập hợp các kết quả từ bảng câu hỏi tùy chọn về mức độ hài lòng trong khóa học được cung cấp trong Bảng 1 . Đối với mỗi câu hỏi được trình bày trong bảng, sinh viên được yêu cầu trả lời theo Thang điểm Likert từ 1 (hoàn toàn không đồng ý) đến 5 (hoàn toàn đồng ý). Kết quả bảng câu hỏi đưa đến nhận định rằng cách tiếp cận hiện tại được sử dụng để giảng dạy về tạo động lực nhóm đã mang đến sự năng động của nhóm, có hiệu quả trong việc tăng sự chú ý của sinh viên đối với chủ đề, tăng số sinh viên tự tin về bản thân mình với nhóm của họ khi bắt đầu các dự án và tăng cường hiểu biết của sinh viên về cách họ và những người khác làm việc theo nhóm.

Bảng 1. Khảo sát mức độ hài lòng của khóa học

\begin{tabular}{|c|c|c|c|c|c|}
\hline \multirow{2}{*}{ Câu hỏi } & \multicolumn{5}{|c|}{ Mức độ hài lòng theo thang điểm Likert } \\
\hline & 1 & 2 & 3 & 4 & 5 \\
\hline Chuẩn đầu ra phù hợp với người học & 0 & 1 & 8 & 35 & 15 \\
\hline Cảm thấy sự năng động sau khi học & 1 & 0 & 24 & 29 & 30 \\
\hline Giảm sự xung đột giữa các cá nhân trong nhóm & 0 & 5 & 15 & 46 & 19 \\
\hline Hiểu sâu hơn về lý thuyết và thực hành thành thạo & 1 & 9 & 20 & 20 & 10 \\
\hline $\begin{array}{l}\text { Hiểu thêm về bản thân và kỹ năng làm việc độc lập, } \\
\text { làm việc nhóm }\end{array}$ & 2 & 2 & 15 & 344 & 27 \\
\hline & 0 & 2 & 10 & 41 & 28 \\
\hline Môn học đáp ứng nhu cầu kiến thức của sinh viên & & & & & \\
\hline Số sinh viên phản hồi ý kiến & 4 & 19 & 92 & 215 & 129 \\
\hline Tỉ lệ phản hồi & $1 \%$ & $4 \%$ & $20 \%$ & $47 \%$ & $28 \%$ \\
\hline
\end{tabular}

\section{Kết luận}

Bài giảng được thực hiện theo chuẩn đầu ra $\mathrm{CDIO}$, mục tiêu của môn học là tập trung vào các giai đoạn triển khai và vận hành trong việc tạo ra một sản phẩm mới hoặc điều khiển một hệ thống. Một mục đích khác là sinh viên chuyên ngành Kỹ thuật Điện - Điện tử nên làm là tìm hiểu kỹ và thực hành với phần cứng công nghiệp một cách thành thạo và có cái nhìn sâu sắc về các khía cạnh thực tế của việc thực hiện các phần thực hành phần cứng. Việc học tập dựa trên bài tập lớn trong phòng thí nghiệm và dự án đã giúp sinh viên có ý 
thức tự giác, tính kỹ luật trong học tập đồng thời mang lại cơ hội phát triển một số kỹ năng kỹ thuật, ví dụ: làm việc nhóm, làm việc độc lập, giao tiếp, tư duy hệ thống, lập kế hoạch thời gian và nguồn lực....

\section{Tài liệu tham khảo}

[1] Åström, K.J. (1999) Automatic Control - The hidden technology. Advances in Control. Highlights of ECC'99. Editor Paul M. Frank. Springer.

[2] Dorf R.C. \& Bishop R.H. (2006). Modern control systems. 11th edition. Pearson, Prentice Hall.

[3] Franklin G.F., Powell J.D. \& Emami - Naeini A. Feedback control of dynamic systems. 6th edition. Pearson, Prentice Hall.

[4] Glad T. \& Ljung L. Reglerteknik (2006). Grundläggande teori. 4th edition. Studentlitteratur. 